\title{
MR imaging characteristics in primary lymphoma of bone with emphasis on non-aggressive appearance
}

\author{
Fenna H. Heyning • Herman M. J. A. Kroon • \\ Pancras C. W. Hogendoorn • Antonie H. M. Taminiau • \\ Henk-Jan van der Woude
}

Received: 19 January 2007 / Revised: 19 March 2007 / Accepted: 23 April 2007 / Published online: 9 June 2007

(C) ISS 2007

\begin{abstract}
Purpose To assess the heterogeneity of magnetic resonance (MR) imaging characteristics in primary lymphoma of bone (PLB), in particular the non-aggressive appearance.

Subjects and methods In a retrospective study, MR imaging features were analyzed in 29 patients with histologically proven PLB. The following parameters were evaluated: tumor size, bone marrow and extension into soft tissues, signal characteristics of bone marrow and soft-tissue components, including enhancement, and involvement of cortical bone (complete disruption, focal destruction, permeative destruction and cortical thickening).

Results PLB presented with extension into the soft tissue in $22(76 \%)$ of 29 patients, was only subtle in three of these 22 patients, and was absent in seven patients. Signal intensity (SI) of the soft-tissue part was most frequently homoge-
\end{abstract}

\section{F. H. Heyning \\ Department of Internal Medicine, \\ Leiden University Medical Center,}

Leiden, The Netherlands

H. M. J. A. Kroon

Department of Radiology, Leiden University Medical Center, Leiden, The Netherlands

P. C. W. Hogendoorn

Department of Pathology, Leiden University Medical Center,

Leiden, The Netherlands

A. H. M. Taminiau

Department of Orthopedic Surgery,

Leiden University Medical Center,

Leiden, The Netherlands

H.-J. van der Woude $(\square)$

Department of Radiology, Onze Lieve Vrouwe Gasthuis, P.O. Box 95500, 1090 HM Amsterdam, The Netherlands

e-mail: h.j.vanderwoude@olvg.nl neously isointense with muscle on T1-weighted images (90\%) and high on T2-weighted images (91\%). Enhancement was predominantly homogeneous and diffuse (82\%). In $93 \%$ of patients cortical bone appeared abnormal: among those patients complete cortical disruption was seen in $28 \%$, with extension into soft tissues in all but one patient; a permeative pattern of destruction was present in $52 \%$ of patients, $66 \%$ of these had an associated soft-tissue mass. Two patients with normal-appearing cortical bone had no extension into soft tissues. In two patients focal cortical destruction was noticed; in one patient cortical bone was homogeneously thickened, and in one patient PLB was selectively localized within the cortical bone. SI of the bone marrow tumor component was more frequently heterogeneous (in 54\%), compared with the soft-tissue component, being high on T2-weighted images in $89 \%$, intermediate in $7 \%$ and low in $4 \%$. Similarly, enhancement was heterogeneous in $59 \%$.

Conclusion The MR imaging appearance of PLB is variable. In $31 \%$ of PLB patients, the tumor was intraosseous, with linear cortical signal abnormalities or even normal-appearing or thickened cortical bone without softtissue mass, and, as such, PLB may not infrequently look non-aggressive on MR imaging.

Keywords Lymphoma $\cdot$ Tumor $\cdot$ Bone $\cdot$ MR imaging

\section{Introduction}

Primary lymphoma of bone (PLB) is a rare disease, accounting for less than $1 \%$ of all non-Hodgkin's lymphoma (NHL) cases and 3-5\% of all extra-nodal lymphoma cases [1-3]. According to the World Health Organization (WHO) classification of hematological malignancies, it is a distinct 
clinico-pathological subtype of extra-nodal, diffuse, large B-cell lymphoma (DLBCL) with a relatively homogeneous pathological and clinical presentation [4, 5]. Almost all tumors are of the large B-cell type, morphologically composed of large centroblastic cells, frequently with multilobated nuclei [5-8]. The prognosis of PLB, depending on staging and histologic classification, is favorable following combined modality (chemo- and radiation) therapy, with a 5 -year overall survival rate that extends to $88 \%$ [7, 9-12].

Debates are ongoing as to whether there is a uniform imaging pattern in PLB, in particular on the magnetic resonance (MR) imaging characteristics of PLB. Some authors have stated that a tumor with normal-appearing cortical bone and a substantial soft-tissue mass is likely to be a non-Hodgkin's lymphoma [13, 14]. One study focused on the hypointense signal intensities on T2-weighted images as a feature that may be characteristic of PLB as opposed to other malignant tumors [15]. In other studies MR findings were found to be non-specific and not useful for differentiating PLB from other small, blue, round cell tumors, including Ewing's sarcoma and small cell osteosarcoma and (in older age groups) tumors such as metastases of carcinoma and plasmacytoma [16]. In addition, the plain radiographic appearance of PLB differs from virtually normal to a severely permeative pattern of bone destruction with a blastic, lytic or mixed appearance, and, as such, is also non-specific [17-19].

The order of differential diagnosis is, therefore, mainly based on the patient's age, race and clinical features.

To assess the homogeneity or heterogeneity of MR imaging characteristics in PLB, and to assess the rate of a non-aggressive MR imaging appearance, we evaluated various features known from the literature, with emphasis on cortical bone manifestation and marrow and soft-tissue signal characteristics, in 29 patients in the present retrospective imaging study.

\section{Subjects and methods}

The files of 29 patients with a histologically proven diagnosis of PLB, and MR imaging studies available at the time of diagnosis, were retrieved from the archive of the Netherlands Committee on Bone Tumors, representing the period between 1989 and 2004. All histological diagnosis were re-assured for this study.

Patients' characteristics

The study group consisted of 29 patients, 14 female and 15 male. In the majority of the patients the tumors were located in the femur $(n=13)$ or humerus $(n=6)$.
Other tumor sites included the ilium $(n=4)$, scapula $(n=2)$, tibia, acetabulum, lumbar vertebra and mandibula (one patient each). The age of the patients ranged from 19-81 years (median 40 years).

\section{MRI}

Since the Netherlands Committee on Bone Tumors serves as a consulting medium for the entire country, MRI studies were performed on different imagers with field strengths between $0.5 \mathrm{~T}$ and $1.5 \mathrm{~T}$. T1-weighted spin echo (SE) sequences were available for 28 patients; TRs varied from 400-800 ms, with TEs from 12-25 ms. T2-weighted (fast) SE sequences were available for 29 patients, with fatselective pre-saturation sequences for 14 patients. TRs varied from 2,000-7,000 ms, with TEs from 70-130 ms. Contrast-enhanced, T1-weighted, SE series after intravenous injection of $0.2 \mathrm{mmol}$ gadopentetate dimeglumine (Gd-DTPA) were available for 25 of 29 patients, with fatselective pre-saturation sequences for ten patients. A dynamic contrast-enhanced sequence was used for only three patients. For all patients a combination of axial and longitudinal (either coronal or sagittal) images was available for evaluation.

Imaging evaluation

The following parameters were evaluated in consensus by two experienced musculoskeletal radiologists:

Tumor size Tumor dimensions were established by measuring length, width and depth, assessed from the combination of axial and longitudinal images.

Extension into the soft tissues Presence or absence of a softtissue component, defined as a mass beyond the margins of cortical bone and periosteum, was determined.

Soft-tissue edema Soft-tissue edema was considered to be present when areas of peri-lesional high signal intensity (SI) were noticed on T2-weighted sequences and/or contrastenhanced T1-weighted sequences in an ill-defined feathery pattern, without disruption of the fascial planes.

Signal intensities of soft-tissue part SIs were defined as predominantly high, intermediate, low or mixed, relative to muscle SI, assessed on both T1- and T2-weighted sequences. Furthermore, homogeneity or heterogeneity of the SI on T2-weighted images was assessed.

Enhancement of soft-tissue part The pattern of tumoral enhancement on T1-weighted Gd-DTPA-enhanced sequences 
(absent, peripheral or diffuse) was recorded, as well as the homogeneity or heterogeneity.

Cortical bone destruction Appearance of the cortical bone was determined as normal or abnormal on both T1- and T2weighted images. Abnormal cortical bone appearance was subdivided into complete cortical destruction (normal low SI, completely replaced by high signal intensity of tumor), focal destruction (normal low signal intensity, partially replaced by high SI of tumor with focal disturbance of cortical integrity), permeative destruction (fine linear pattern of intracortical high SI, without disturbance of cortical integrity) or cortical thickening (thickening of cortical bone, without signal abnormalities).

Bone marrow involvement SIs of the bone marrow component and homogeneity were assessed on T1- and T2weighted sequences, compared with muscle SI (higher, equal/intermediate, lower, mixed) and opposed to the high SI of normal bone marrow. Tumor margins were defined as sharp or unsharp.

Enhancement of bone marrow component The presence or absence of intra-osseous tumoral enhancement and the pattern (homogeneous, heterogeneous, focal, diffuse) of enhancement on static T1-weighted Gd-DTPA-enhanced sequences were determined.

Histological classification and immunohistochemistry The pathology diagnosis was established according to the World Health Organization classification on biopsy material, using standard histological criteria and immunohistochemistry using antibodies directed against vimentin, CD45, CD3,
CD20, CD79a, and CD99. Immunohistochemical staining was performed on $4 \mu \mathrm{m}$ sections of formalin-fixed, paraffin-embedded tissues, using standard procedures. In this study, all tumors were of the large B-cell type.

\section{Results}

Tumor size The mean maximum tumor diameter was $11 \mathrm{~cm}$ (range $3-18 \mathrm{~cm}$ ).

Extension into the soft tissues Tumor extension into the soft tissues was apparent in 22 of the 29 patients (Fig. 1) (76\%), in three of these patients this was very discrete (Fig. 2). In the remaining seven patients $(24 \%)$, the tumor was entirely confined to the intramedullary and/or cortical compartment (Fig. 3).

Soft-tissue edema Peri-lesional soft-tissue edema was encountered in approximately half of the patients (15 out of $29,52 \%$ ), whereas, in the other 14 patients, it was absent.

Signal intensities of soft-tissue part Appearance on T1weighted sequences could be assessed for 21 patients bearing tumors with extension into the soft-tissues: in 19 $(90 \%)$ of these 21 patients the SI was intermediate and equal to that of muscle. In two patients the SI was higher than for muscle. In 22 patients the SI on T2-weighted sequences could be assessed, being high (higher than that of muscle, lower than that of fat) in 20 patients (91\%). This high signal was predominantly homogeneous in 18 of those 20 patients and heterogeneous in the other four.
Fig. 1 An 80-year-old male patient with primary nonHodgkin's lymphoma of the proximal humerus. Axial T1weighted turbo-spin echo (TSE) (TR/TE 575/15 ms) images before (a) and after (b) Gd-DTPA administration, the latter sequence with fat-selective presaturation. Intra-osseous tumor with circumferential substantial extension into the soft tissue. There is partly complete cortical disruption of cortical bone and otherwise permeative destruction shown as a fine linear pattern of abnormal increased cortical SI (a), even more conspicuous on contrast-enhanced images (b, arrow)
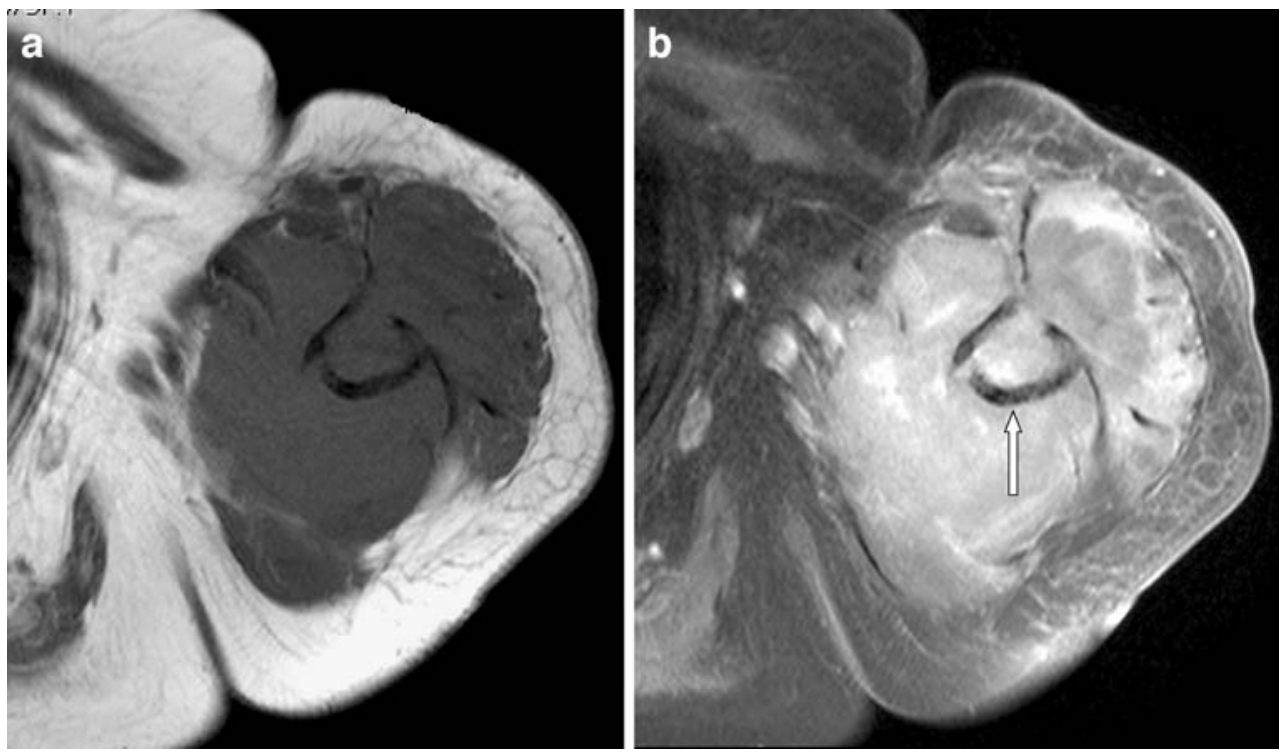
Fig. 2 A 32-year-old female patient with primary nonHodgkin's lymphoma of the distal femur diaphysis. Axial T2-weighted fat-suppressed turbo-spin echo (TSE) (TR/TE $3799 / 80 \mathrm{~ms}$ ) image (a) and axial T1-weighted contrast-enhanced fat-suppressed TSE (TR/TE 500/15 ms) image (b). Heterogeneous high SI (a) of intraosseous component and limited circumferential soft-tissue cuff. Heterogeneous enhancement. Permeated cortical bone with linear intracortical increased SI, particularly seen on Gd-enhanced images (b, arrow)


Enhancement of soft-tissue part Enhancement of the softtissue component of the tumor could be determined in 17 patients; for the other 12 patients contrast-enhanced studies were not available. Enhancement of the soft-tissue part occurred in all evaluable patients, being predominantly homogeneous and diffuse in $14(82 \%)$ patients and heterogeneous in the other three patients (Fig. 1).

Cortical bone destruction For two of the 29 patients the entire cortical bone was scored as normal. In those two patients there was also no extension into soft-tissue (Fig. 3). In 27 patients (93\%) the cortical bone was abnormal. A permeative cortical destruction pattern was noticed for a small majority of patients (15 of $29,52 \%)$. In ten of these 15 patients, an associated soft-tissue mass was apparent (Fig. 4); however, in two patients, this was absent. In the remaining three patients only limited extra-cortical abnormalities were present (Fig. 2). Complete cortical disruption was found in eight patients (Fig. 1) (28\%). All but one of these eight patients had significant extension into the softtissue. Focal destruction was seen in two patients, and homogeneous cortical thickening in one patient. Moreover, selective intracortical tumor localization was encountered in one other patient.

Bone marrow involvement Signal intensity of the intraosseous tumor component on T1-weighted images could be assessed in 26 patients being predominantly intermediate (equal to muscle) in 25 patients (Figs. 1, 4) and predominantly low (lower than muscle) in one patient. In three patients $(12 \%)$ of the former group, a combination of intermediate and low signal intensity was found. Demarcation in the longitudinal extent was scored as sharp in 11 and shaded in nine patients. On T2-weighted images (available in 28 patients) the SI of the bone marrow compartment was high in 25 patients $(89 \%)$, intermediate in two patients $(7 \%)$
Fig. 3 A 26-year-old female patient with primary nonHodgkin's lymphoma of the distal femur. Sagittal (a) and axial (b) T1-weighted contrast-enhanced fatsuppressed turbo-spin echo (TSE) (TR/TE 600/20 ms) images. There is inhomogeneous enhancement of the intraosseous tumor, with normal appearing cortical bone and, besides enhancing periosteum, no associated soft tissue mass
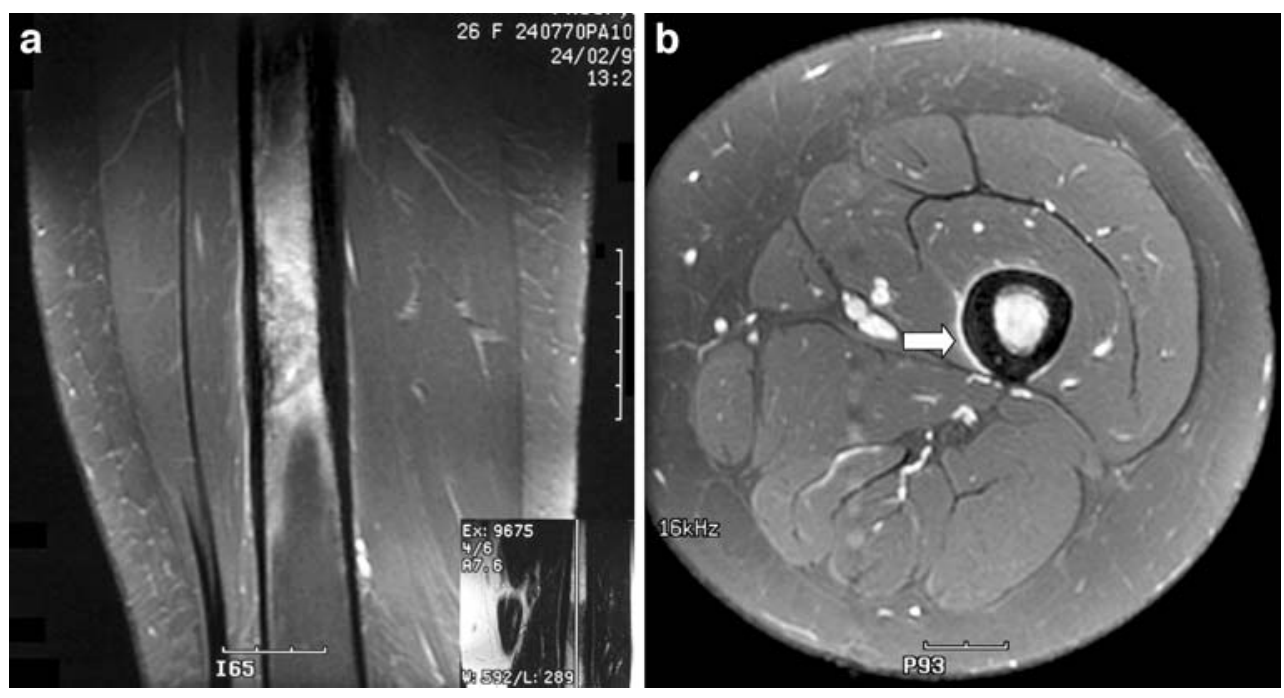

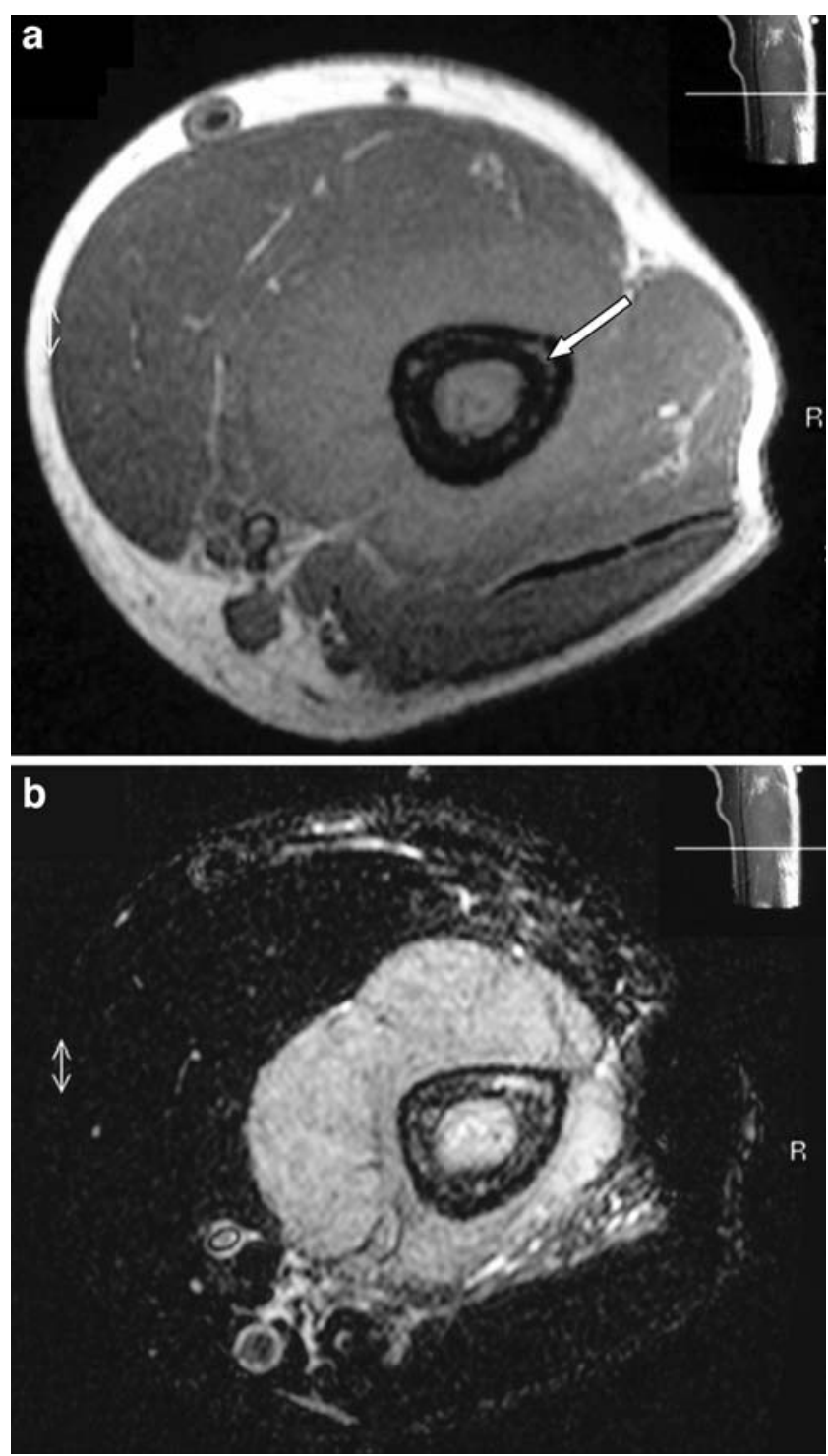

Fig. 4 A 58-year-old male patient with primary non-Hodgkin's lymphoma of the distal humerus. Axial T1 turbo-spin echo (TSE) (TR/TE 504/13 ms) (a) and T2-weighted TSE (5,927/80 ms) image with fat-selective pre-saturation (b). Primary intra-osseous tumor with circumferential soft-tissue mass, homogeneously isointense on T1 and high on T2-weighted sequences. Normal cortical thickness with discrete signal abnormalities on $\mathrm{T} 1$ (a, arrow), more conspicuous on T2-weighted sequences (b)

and low in one patient. The SI of the bone marrow tumor component was homogeneous in 13 of 28 patients $(46 \%)$, however, inhomogeneous in 15 patients (54\%). (Figs. 2, 5).

Enhancement of bone marrow component Enhancement of the bone marrow tumor component could be assessed in 23 patients. No enhancement was noticed in only one patient. The other 22 patients showed enhancement, which was homogeneous in nine patients $(41 \%)$, and heterogeneous in 13 patients (59\%) (Figs. 2, 3, 5).

\section{Discussion}

Primary non-Hodgkin's lymphoma of bone (PLB) is a rare entity among musculoskeletal tumors, and it is also uncommon among extra-nodal sites of NHL. The morphology of these lymphomas is identical to that of lymphomas at other sites, but the clinical course is often more protracted. Prognosis of PLB depends mainly on staging and on histological classification, but, generally, the clinical outcome, after treatment with combined chemo- and radiation therapy, is favorable, with a 5-year overall survival rate rising to $88 \%[1-5,9-11,18,20]$. On the other hand, Lewis et al. reported a 5 -year survival rate of $58 \%$ and no statistically significant difference between PLB and systemic bone lymphoma [6]. With regard to histological subtype, tumor localization and clinical presentation, patients with PLB display homogeneous clinicopathological features $[4,5]$. Almost all tumors are localized in long bones (particularly the femur, tibia, pelvis and humerus), and patients present with pain and/or a palpable mass. The literature describes a significant male preponderance, which is not confirmed in this present study. Although PLB presents in a wide age range of patients, evenly distributed among the second through eighth decades of life, in general, it is more frequently encountered in patients over 30 years of age, with a peak incidence in the fifth decade, in contrast to that of Ewing's sarcoma and osteosarcoma $[6,8,10,11,13$, $14,18]$. In the latter patient group, a significant decrease in incidence is found beyond the third decade. In our study group of 29 patients a broad range of ages at presentation was also found (19-81 years); the median age was 40 years.

Plain radiographs of PLB often show destructive bone lesions, generally with periosteal layering and cortical disruption. On the other hand, plain radiographs of PLB may appear virtually normal or non-specific and can be overlooked as being normal. As such, there is no specific conventional radiographic pattern, and the radiographic differential diagnosis of PLB includes a broad spectrum of both benign (including osteomyelitis) and malignant (such as osteosarcoma, Ewing's sarcoma) lesions [8, 16-19]. These tumors and tumor-like lesions may demonstrate similar radiographic features but warrant different types of therapy. In this respect PLB is associated with a better prognostic outcome than that for other small round cell tumors. Literature on the presentation of MR imaging features of PLB is relatively scarce and contradictory. These studies, frequently with limited numbers of patients, mainly focused on the appearance of the cortical bone, relative to associated soft-tissue abnormalities, and on SI characteristics [13-16, 18, 19, 21, 22]. Low SI on T2-weighted sequences has been mentioned as a feature of PLB, probably due to a high content of fibrous tissue [15]. We could not confirm this feature to a great extent in our 
Fig. 5 A 29-year-old male patient with non-Hodgkin's lymphoma of the proximal humerus. Axial T1-weighted contrast-enhanced (TR/TE 550/12 ms) (a) and T2-weighted (b) turbo-spin echo (TSE) (TR/TE 3,021/

$80 \mathrm{~ms}$ ) images with fat-selective pre-saturation. Intra-osseous tumor localization with no extension into soft-tissue and heterogeneous high SI on both sequences
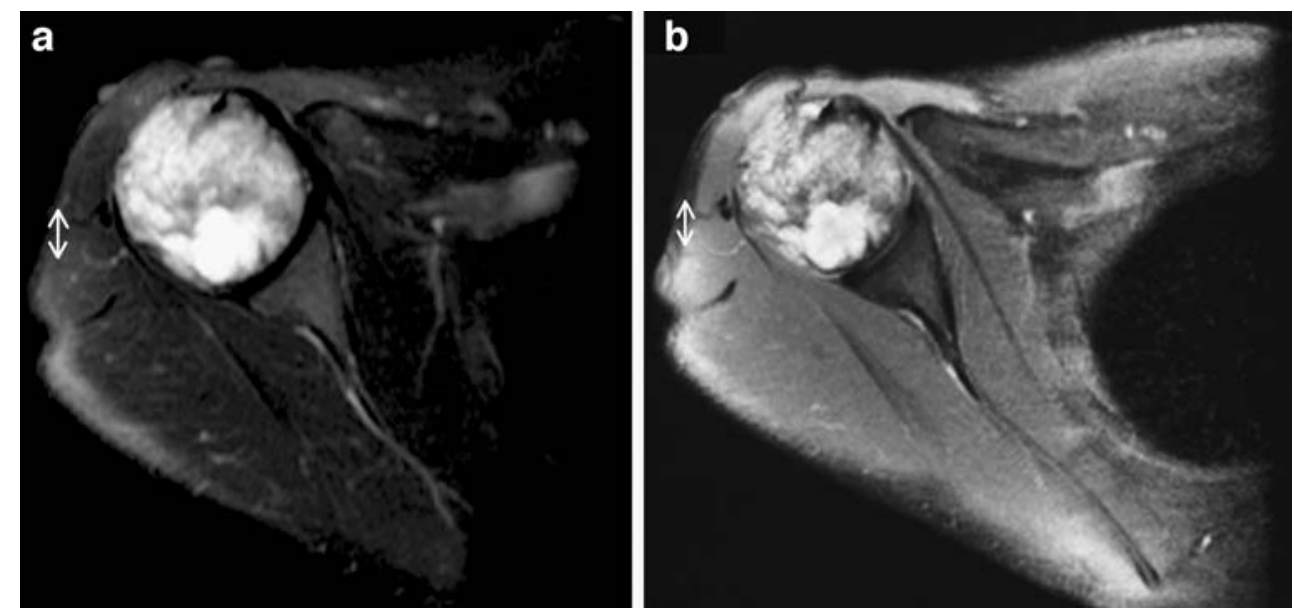

population. Low SI on T1- or T2-weighted sequences was present in only one patient each. Almost all tumors showed a non-specific combination of intermediate (equal to that of muscle) SI on T1-weighted sequences and high SI on T2weighted sequences.

Several studies on PLB and MR imaging focused on the pattern of cortical destruction in order to use it as a differentiating criterion. It was stated that a tumor with a normal (appearing) cortex and a substantial soft-tissue mass is likely to be lymphoma $[13,14]$. MR imaging may show small linear foci of intermediate or high SI on T2-weighted sequences, penetrating the cortical bone. This MR pattern of permeative cortical destruction is, however, not specific to lymphoma. It can be seen in other small round cell tumors (in the younger age group) as well as in different metabolic and inflammatory processes, including hyperparathyroidism and osteomyelitis and, for instance, myeloma or metastatic carcinoma (in the older age group) $[13,14]$. In our study, cortical abnormalities were present in $93 \%$ of patients, and extension into the soft tissue was found in $76 \%$, but various types of destruction and combinations with or without such extension were recognized (Table 1). A pattern of permeative cortical destruction with linear foci of abnormal SI was noticed in 15 of the 29 (52\%) patients. Ten of these 15 patients had a definite soft-tissue mass. In five patients, however, this was minimal or even absent. In $28 \%$ of our patient population complete cortical disruption could be appreciated; in all but one patient it was accompanied by a large soft-tissue mass. In another two patients, the cortical bone appeared to be completely normal, and in these patients there was no associated softtissue mass. In one patient with histologically confirmed PLB the tumor was selectively located within the cortical bone, without further extension into the soft tissue and/or bone marrow involvement. In one patient the cortical bone was homogeneously thickened, a feature that can also be encountered in reactive processes (after trauma or infection). Peri-osteal or cortical lymphoma without medullary involvement is a very rare manifestation of NHL, but it has been previously described [23]. In this respect, it can be concluded that in patients with PLB the pattern of cortical destruction is heterogeneous and non-specific.

MR imaging is the optimal modality to demonstrate the extent of bone marrow replacement by PLB, particularly using T1-weighted sequences [14, 24]. Multi-centric bone marrow disease is likely to be detected by longitudinal series with a large field of view. Signal intensities of the bone marrow component were non-specific in the present series, being intermediate on T1-weighted sequences and high on T2-weighted sequences in almost all patients. In
Table 1 Relationship between MR imaging appearance of cortical bone and extension into the soft tissue in 29 patients with PLB

\begin{tabular}{lllll}
\hline Cortex appearance & $\begin{array}{l}\text { Extension into } \\
\text { soft-tissue substantial }\end{array}$ & $\begin{array}{l}\text { Extension into } \\
\text { soft-tissue limited }\end{array}$ & $\begin{array}{l}\text { Extension into } \\
\text { soft-tissue absent }\end{array}$ & Total \\
\hline Normal & 0 & 0 & 2 & 2 \\
Permeative destruction & 10 & 3 & 2 & 15 \\
Focal disruption & 2 & 0 & 0 & 2 \\
Complete disruption & 7 & 0 & 1 & 8 \\
Cortex thickening & 0 & 0 & 1 & 1 \\
Tumor within cortex only & 0 & 0 & 1 & 1 \\
Total & 19 & 3 & 7 & 29 \\
\hline
\end{tabular}


only three patients a combination of intermediate and low signal intensity was found. Demarcation in the longitudinal extent was scored as sharp in 11 and unsharp in nine patients. The SI of the bone marrow tumor component was homogeneous in 13 of 28 patients (46\%); however, it was inhomogeneous in 15 patients $(54 \%)$, which can be explained by the intermingling of normal bone marrow between areas of tumor. Contrast-enhanced images of the bone marrow showed a similar distribution; enhancement was diffuse and homogeneous in $41 \%$ and heterogeneous in $59 \%$ of the patients whose condition was eligible for evaluation. Opposed to the bone marrow component, the soft- tissue component, if present, was far more homogeneous, both on T1-weighted contrast-enhanced images $(82 \%)$ and as high SI on T2-weighted images (82\%).

The data obtained in this retrospective study demonstrate that, in contrast to the relatively homogeneous clinical and pathological presentation, the MR imaging features are not uniform in patients with PLB. Although the majority of our patients displayed a combination of definite cortical abnormalities (either permeative destruction or complete disruption) and extension into soft-tissue, lymphoma may show different appearances. Particularly, intra-osseous lesions with linear cortical signal abnormalities without substantial soft-tissue mass, or even normal-appearing or thickened cortical bone without soft-tissue mass, may look nonaggressive and even benign. In the present study no fewer than nine of 29 patients (31\%) with PLB presented in that manner. Radiologists should thus be aware of that in the right clinical context.

There are several limitations concerning this retrospective and largely descriptive study. The data obtained were collected from various institutions that had used different MR imaging systems, field strengths and protocols. In order to collect a larger number of patients, given the rarity of PLB, we could not avoid this. The assessment of heterogeneity or homogeneity of signal characteristics is quite subjective; however, for the goal of this study it was of minor importance. Fast dynamic contrast-enhanced sequences were used only incidentally. Nowadays, these acquisitions are widely used to assess the biologic behavior of primary bone and soft-tissue tumors and to determine the response to chemo- and/or radiation therapy. With regard to PLB, the rapid decrease of tumor volume and the complete disappearance of the soft-tissue component on MR imaging has been described as a significant indicator of good response to treatment [24]. In this respect, MR imaging has a direct impact on individual patient care, which is however, outside the scope of this study. We could not determine how MR imaging had caused any change in clinical approach in the patients with PLB described here. Differentiating PLB from other malignant small round cell tumors, on the one hand, or reactive processes, on the other hand, based on (MR) imaging criteria only, is, in our opinion, not possible and not useful, which stresses the need for accurate core biopsy, which is definitely crucial for both diagnosis and staging purposes.

\section{References}

1. Huvos AG. Skeletal manifestations of malignant lymphomas and leukemias. In: Bone tumors: diagnosis, treatment, and prognosis. Philadelphia: Saunders; 1979.

2. Mulder JD, Kroon HM, Schutte HE, Taconis WK. Non-Hodgkin's lymphoma. In: Radiologic atlas of bone tumors. Amsterdam, The Netherlands: Elsevier; 1993.

3. Mirra JM, Picci P, Gold RH. Skeletal manifestations of malignant lymphomas and leukemias. In: Bone tumors: clinical, radiologic and pathologic correlations. Philadelphia, PA: Lea and Febiger; 1989.

4. Fletcher CD, Unni KK, Merlens F. WHO classification of tumours. Pathology and genetics of tumours of soft tissue and bone. Lyon: IARC press; 2002.

5. Heyning FH, Hogendoorn PCW, Kramer MHH, et al. Primary non-Hodgkin's lymphoma of bone: a clinicopathologic investigation of 60 cases. Leukemia 1999; 13: 2094-2098.

6. Lewis V, Primus G, Anastasi J, et al. Oncologic outcomes of primary lymphoma of bone in adults. Clin Orthop Relat Res 2003; 415: 90-97.

7. Barbieri E, Cammelli S, Mauro F, et al. Primary non-Hodgkin's lymphoma of the bone: treatment and analysis of prognostic factors for stage I and stage II. Int J Radiat Oncol Biol Phys 2004; 59: 760-764.

8. de Camargo O, dos Santos Machado T, Croci A, et al. Primary bone lymphoma in 24 patients treated between 1955 and 1999. Clin Orthop Relat Res 2002; 397: 271-280.

9. Vasallo J, Roessner A, Vollmer E, Grundmann E. Malignant lymphomas with primary bone manifestation. Pathol Res Pract 1987; 182: 381-389.

10. Baar J, Burkes RL, Bell R, Blackstein ME, Fernandes B, Langer F. Primary non-Hodgkin's lymphoma of bone. A clinicopathologic study. Cancer 1994; 73: 1194-1199.

11. Beal K, Allen L, Yahalom J. Primary lymphoma of bone: treatment results and prognostic factors with long-term follow-up of 82 patients. Cancer 2006; 106: 2652-2656.

12. Baar J, Burkes L, Gospodarowicz M. Primary non-Hodgkin's lymphoma of bone. Semin Oncol 1999; 26: 270-275.

13. Mulligan ME, McRae GA, Murphey MD. Imaging features of primary lymphoma of bone. AJR Am J Roentgenol 1999; 173: 1691-1697.

14. Krishnan A, Shirkhoda A, Tehranzadeh J, Armin AR, Irwin R, Les K. Primary bone lymphoma: radiographic-MR imaging correlation. Radiographics 2003; 23: 1371-1383.

15. Stiglbauer R, Augustin I, Kramer J, Schurawitzki H, Imhof H, Radaszkiewicz. MRI in the diagnosis of primary lymphoma of bone: correlation with histopathology. J Comput Assist Tomogr 1992; 16: 248-253.

16. Hicks DG, Gokan T, O'Keefe RJ, et al. Primary lymphoma of bone; correlation of magnetic resonance imaging features with cytokine production by tumor cells. Cancer 1995; 75: 973-980.

17. Mendenhall NP, Jones, Kramer BS, et al. The management of primary lymphoma of bone. Radiother Oncol 1987; 9: 137-145.

18. Manaster BJ. Primary bone lymphoma: radiographic-MR imaging correlation. Invited commentary. Radiographics 2003; 23: 13841386. 
19. Clayton F, Butler JJ, Ayala A, Ro JY, Zornoza J. Non-Hodgkin's lymphoma in bone: pathologic and radiologic features with clinical correlates. Cancer 1987; 60: 2494-2501.

20. Yuste A, Segura A, Lopez-Tendero P, Girones R, Montalar J, Gomez-Codina J. Primary lymphoma of bone: a clinico-pathologic review and analysis of prognostic factors. Leuk Lymphoma 2004; 45: 853-855.

21. Haussler MD, Fenstermacher MJ, Johnston DA, Harle TS. MRI of primary lymphoma of bone: cortical disorder as a criterion for differential diagnosis. J Magn Reson Imaging 1999; 9: 93-100.
22. Salter M, Sollacio RJ, Bernreuter WK, Weppelmann B. Primary lymphoma of bone: the use of MRI in pretreatment evaluation. Am J Clin Oncol 1989; 12: 101-105.

23. Campbell S, Filzen T, Bezzant S, et al. Primary periosteal lymphoma: an unusual presentation of NHL. Skeletal Radiol 2003; 32: 231-235.

24. Mengiardi B, Honegger H, Hodler J, Exner U, Csherhati M, Bruhlmann W. Primary lymphoma of bone: MRI and CT characteristics during and after successful treatment. AJR Am J Roentgenol 2005; 184: 185-192. 